\title{
GEOENGINEERING CLIMATE CHANGE: TREATING THE SYMPTOM OVER THE CAUSE?
}

\author{
An Editorial Comment
}

The present article by Crutzen (2006) on the use of albedo enhancement to address the problem of greenhouse warming is bound to evoke diverse reactions among the community. It is important to note that Crutzen argues that this idea be studied in depth and openly before any large scale action is taken. A basic assumption to this approach is that we, humans, understand the Earth system sufficiently to modify it and 'know' how the system will respond. Cicerone in his article argues that open discussion of these ideas is an appropriate means to explore engineering solutions to climate change, and further proposes a protocol to prevent inadvertent abuse of geoengineering experiments. These papers evoke both scientific and ethical issues that should stimulate discussions on the engineering of Earth's climate system.

We have already 'chosen' to geoengineer our climate system through our use of fossil fuels, where the engineering of the climate system is an inadvertent by product of our values around forms of consumption. Proposals to consciously alter the climate system to treat the symptom of our behaviors imply we understand all of the complexities of Earth as a system. At times Earth performs a stratospheric albedo enhancement experiment through the eruption of volcanoes. As pointed out by Crutzen, the Earth does cool due to this experiment, but this experiment also provides ample evidence of the non-local and non-linear response of Earth's climate system, e.g. winter NH warming. This example exhibits how Earth's climate system is far more complex than a simple energy balance picture. For this reason, I support Crutzen's argument that more detailed and comprehensive modeling studies be carried out with regards to experiments. But my concern is that all models have their limitations (e.g. note the inability of models to predict the appearance of the Antarctic ozone hole before it was observed). When will we know a model is 'good enough' to go out and perform a real experiment?

On the issue of ethics, I feel we would be taking on the ultimate state of hubris to believe we can control Earth. We (the industrially developed world) would essentially be telling the (rest of the) world not to worry about our insatiable use of energy. In essence we are treating the symptom, not the cause. Our species needs to begin to address the cause(s) behind the problem. For example, an analysis of the U.S. contribution to $\mathrm{CO}_{2}$ emissions indicates that these emissions in part arise from three factors: the large number of SUVs, the size of homes, and distance we drive to work. I would argue that the first two of these factors are ones of choice, and not necessity. Yet, the American public chooses to buy SUVs and build large 
homes. Why? It seems that we need to address the fundamental issue of value, before tinkering with a system that we do not completely understand.

I recognize that Crutzen's proposal comes from his deep concern for Earth. I also recognize his concern arises from our species reluctance to address the cause(s) of our dilemma. But I feel that treating the cause(s) rather than the symptom is the more appropriate approach to the problem. However, as a scientist, I also recognize the importance of exploration of ideas, and that open dialogue and study of this issue (as proposed by Crutzen and seconded by Cicerone) is an important part of Earth studies. Let the dialogue begin...

\section{Reference}

Crutzen, P. J.: 2006, 'Albedo enhancement by stratospheric sulfur injections: A contribution to resolve a policy dilemma?', Climatic Change, this issue, DOI: 10.1007/s10584-006-9101-y.

National Center for Atmospheric Research

JEFFREY T. KIEHL

P.O. Box 3000 Boulder

CO 80307

E-mail:jtkon@vcar.edu 